\title{
Uma proposta de Modelagem Analítica para o uso efetivo de Atividades Complementares no Ensino Superior
}

\author{
Leandro A. Silva, Fábio Silva Lopes, Arnaldo Rabello de Aguiar Vallim Filho
}

Faculdade de Computação e Informática - Universidade Presbiteriana Mackenzie Rua da Consolação, 930 - 01302-907 - São Paulo - SP - Brasil

\{leandroaugusto.silva, flopes, aavallim\}@mackenzie.br

\begin{abstract}
Resumo. Atividades Complementares representam uma exigência do Ministério da Educação com o intuito de ampliar a formação dos alunos de cursos superiores. Estas atividades se constituem em requisitos obrigatórios dos cursos de graduação, representando até 20\% da carga horária desses cursos. Neste trabalho, apresentamos uma modelagem analitica para auxiliar o uso efetivo deste instrumento que é a complementação do aluno através de conceitos de Inteligência de Negócios e Mineração de Dados. Os resultados apresentados por meio de painéis permitem analises na visão do gestor como a quantidade de horas para temáticas de interesses dos alunos e para turmas de alunos; $e$ analises na visão do aluno como a quantidade de horas realizadas e a comparação com a média da turma. Estes resultados permitem futuramente analisar complementação de conceitos, defasagem do curso e até mesmo controlar evasão de estudantes.
\end{abstract}

\begin{abstract}
Complementary Activities are requirements of the Federal Secretary of Education that aims to expand the training given to higher education students. These activities constitute mandatory requirements for undergraduate courses, representing up to $20 \%$ of the workload of such courses. In this paper, we introduce an analytical modeling to assist the effective use of this instrument through the concepts of Business Intelligence and Data Mining. The results presented through dashboards allow analysis in the manager view as the amount of hours for topics and for classrooms and analyzes the student vision as the number of hours performed and compared to the class average. In the future these results allow analyze future topics for supplementary concepts, gaps in the course or student retention.
\end{abstract}

\section{Introdução}

Segundo o Ministério da Educação (MEC), "As Atividades Complementares têm a finalidade de enriquecer o processo de ensino-aprendizagem, privilegiando a complementação da formação social e profissional" [MEC_A, 2013]. Esta exigência do MEC foi posta em prática, de forma efetiva no $1^{\circ}$ Semestre de 2009, em uma Faculdade de Computação de uma grande Universidade Pública Brasileira, aqui denominada por apenas Unidade Universitária (UU), e que é o foco deste estudo.

As Atividades Complementares (AC), nesta UU, são gerenciadas por um Coordenador, que tem como papel principal preparar um catálogo de modalidades; receber e avaliar solicitações de atividades; e atribuir horas aos alunos. Além disso, o coordenador também atua na divulgação de atividades, auxilia em eventos oferecidos 
pela UU e também pela Universidade, em prol de oferecer uma maior diversidade de atividades aos alunos.

Além dos requisitos comuns em cursos de Computação como cumprimento de carga horária e horas de estágio, nesta UU, os alunos devem cumprir 200 horas de AC para estarem aptos a colar grau. Este número total de horas é calculado com base na carga horária do curso. As diretrizes do MEC [MEC_B, 2013] fazem uma recomendação para que seja inferior a $20 \%$ da carga horária total do curso.

Por ser uma exigência, as $\mathrm{AC}$ geram uma carga administrativa intensa às Instituições de Ensino Superior (IES) para o cumprimento desse quesito. Para se mensurar este trabalho, atualmente a UU oferece dois cursos de bacharelado: Ciência da Computação (CC) e Sistemas de Informação (SI), um curso de licenciatura: Matemática (Mat) e um curso Superior de Tecnologia em Análise e Desenvolvimento de Sistemas (TADS). O total de alunos matriculados em todos os cursos atinge algo em torno de 1500 alunos, e recebe, anualmente, em média, 360 novos alunos e, em média, 200 alunos se formam a cada ano. É importante ressaltar que na Universidade à qual esta UU pertence, ela não é considerada uma unidade de ensino com muitos alunos - embora estes números sejam, muitas vezes, próximos da quantidade de alunos de Faculdades e Centros Universitários - mas o trabalho que se despende para coordenar as AC é bastante volumoso, principalmente em relação aos formandos.

$\mathrm{Na}$ UU, as atividades consideradas para a quantificação são diversificadas em 22 modalidades, que possibilitam ao aluno um total de 1.020 horas. As modalidades estão distribuídas na tríade de Ensino, Pesquisa e Extensão. No entanto, o que se pretende discutir neste trabalho é uma melhor adequação das modalidades de AC para atuar de forma mais efetiva como uma ferramenta com potencial de ultrapassar os limites de seu papel de "complementar" os estudos durante o período em que o aluno está matriculado no curso. Nesse aspecto, discute-se uma proposta de modelagem analítica dos dados gerados pelas solicitações de atividades complementares, de forma a gerar indicadores que proporcionam o acompanhamento das atividades nos aspectos de monitoramento gerencial e administrativo. Como componente de contribuição deste trabalho, nesta modelagem combinaremos conceitos usados em Inteligência de Negócios ou BI (do inglês, Business Intelligence) [Turban et al., 2009] e Mineração de Dados ou DM (do inglês Data Mining) [Tan et al., 2009], como explicaremos adiante na metologia do trabalho. Estes resultados serão obtidos por meio de painéis (em inglês, dashboards), os quais futuramente permitirão analisar:

- Complementação de conceitos;

- Defasagem do Curso em relação à Tecnologia;

- Formação Pessoal;

- Monitoramento de Atividades;

- Controle de Evasão.

Além desta introdução, que tem o objetivo de contextualizar a problemática estudada e apresentar o objetivo do trabalho, o artigo está dividido como segue. $\mathrm{Na}$ seção 2 é discutida a importância da complementação curricular. $\mathrm{Na}$ seção 3 é apresentado o entendimento das Atividades Complementares na forma de instrumento de auxílio em Instituição de Ensino Superior (IES). Na seção 4 são discutidos os 
conceitos de analises de dados. Já na seção 5 apresenta-se a metodologia experimental, a modelagem proposta e os resultados experimentais. Por fim, na última seção, são apresentadas as discussões finais.

\section{Complementação Curricular}

A complementação curricular se torna necessária uma vez que certos gaps encontrados para a boa formação do aluno transcendem questões ligadas às novas tecnologias (em particular em cursos da área de computação), a temas que eventualmente não são abordados nos cursos, e até mesmo a aspectos comportamentais.

Como abordado por Bittencourt e Figueiredo (2003), a velocidade com que as inovações tecnológicas acontecem não é a mesma na atualização de projetos pedagógicos, sobretudo na área da Computação. Há alguns anos, como exemplo, não existia o conceito de rede social. Esse fenômeno tem provocado mudanças de paradigmas que vão além da troca de mensagem e causam repercussões nas principais disciplinas de Computação como: Linguagens de Programação, Banco Dados, Redes de Computadores e Ambientes Operacionais. E ainda, a recuperação da informação, uma área de conhecimento que apresenta novidades com pouca regularidade, atualmente reúne esforços para melhorar mecanismos de recomendação, baseado em busca de informação não estruturada nas redes sociais e nos sistemas de e-commerce. Tal cenário sugere atenção para os currículos de referencia, no sentido de manter os conteúdos ofertados na graduação em constante atualização. No entanto, não é objeto de discussão deste trabalho e nem ponto de vista dos autores querer substituir disciplinas e conceitos que podem ser considerados como básicos e, portanto fundamentais para $\mathrm{o}$ entendimento de novos conceitos que emergem. O que se pretende discutir é como suprir eventuais gaps e oferecer aos egressos uma formação atualizada com as novidades do mercado de trabalho com o apoio de AC.

No âmbito de pesquisa, as Instituições de Ensino Superior (IES) tem o recurso da Iniciação Cientifica, uma atividade que complementa a formação do estudante, indo além dos conteúdos estudados nas disciplinas do curso. Porém, dependendo do perfil da Instituição (principalmente as particulares) e dos próprios alunos, existe uma forte tendência de que estes alunos queiram ir, cada vez mais cedo, para o mercado de trabalho - que carece cada vez mais de profissionais de Computação -, impulsionados pela possibilidade de autonomia financeira. Assim, torna-se difícil para o aluno, que embora possa ter o interesse, realizar esta atividade. Nesta UU, apenas cerca de $5 \%$ (em média, 10 alunos por semestre) dos alunos realizam atividade de Iniciação Científica. Vale lembrar que os valores pagos pelas bolsas de Iniciação Científica não conseguem concorrer com os valores de estágio que em média é quase três vezes maior que o valor de uma bolsa.

Em termos mais gerais, existem outros aspectos não técnicos que são pouco tratados pelas IES. Como exemplo, questões ligadas a comportamento, tais como a ética e postura que um aluno deve ter em uma entrevista, reuniões, trabalhos colaborativos, entre outros, que são abordadas informalmente nas disciplinas, sem um rigor mais acadêmico, como os demais temas e, portanto, são desafios que na grande maioria dos casos ficam a cargo do próprio aluno que acaba adquirindo esta formação no mercado de trabalho. 


\section{Atividades Complementares}

Na seção anterior, foram discutidas algumas preocupações que a UU em estudo tem sobre a formação do egresso. Discutiu-se sobre a defasagem das IES sobre a tecnologia, formação profissional e formação pessoal. Contudo, neste trabalho, os desafios de como tratar as AC como um recurso para minimizar todas estas preocupações são discutidos.

No catálogo de Categorias de Atividades estão cursos de atualização (técnicos ou de idiomas), monitorias, iniciações cientifica, publicação, participação em eventos científicos, certificação profissional, entre outras. Ao todo são mais de 20 atividades para o aluno escolher. No entanto, há mecanismos de controle que exigem a diversificação de Atividades, ou seja, o aluno não consegue fazer 200 horas apenas com cursos de atualização.

Outro aspecto que tem ajudado em analises de AC para uso de forma mais efetiva está o Sistema de Gerenciamento das Atividades Complementares - GAC [Silva e Vallim, 2011]. Toda solicitação e avaliação são feitas através de um sistema. Isso quer dizer que o aluno entrega um certificado de forma digital, descreve a atividade e o coordenador, por sua vez, avalia a atividade deixada. Havendo necessidade de esclarecimentos, usa-se o mesmo sistema e assim, se estabelece certa interação entre aluno-coordenador.

Todas as solicitações ficam armazenadas em banco de dados e o Coordenador, a cada semestre, usa dessa base para preparar relatórios de solicitações, permitindo-se assim: acompanhamento sobre os números totais de solicitações, situação dos alunos que estão ingressando no último semestre, etc [Silva e Vallim, 2011]. Estes relatórios, ainda, podem servir como indicadores para uso em estratégias de melhor aproveitamento de AC.

Diante do acompanhamento constante das atividades realizadas, as coordenações de Atividades Complementares e de Cursos têm pensado em algumas estratégias para aproveitar melhor este instrumento e os discutem a seguir junto aos conceitos envolvidos para uma modelagem analítica.

\section{Analise de Dados}

Os dados armazenados em formato digital permitem uma serie de analises para tornarmos as Atividades Complementares um mecanismo efetivo na complementação de conceitos, mitigação de deficiências em projetos pedagógicos, monitoramento de atividades e até mesmo o controle de evasão. De forma genérica, chamamos isto de Modelagem Analítica, pois envolvem conceitos de Armazém de Dados, Processamento Analítico On-line, Inteligência de Negócios e Mineração de Dados. Nesta seção apresentaremos uma introdução destas tecnologias para que, na seção seguinte, seja possível a explicação de como elas interagem na metodologia proposta e entendimentos dos resultados experimentais.

\subsection{Inteligência de Negócios}

Inteligência de Negócios ou simplesmente BI (do inglês, Business Intelligence) é uma maneira de apresentar informação facilmente interpretável por um tomador de decisão [Turban et al, 2009]. Isto é feito geralmente por painéis (dashboards em inglês) que permitem o monitoramento de variáveis na forma de gráficos. 
As variáveis dos painéis geralmente são extraídas de uma base de dados analítica. Isto é, a partir de uma ou mais bases para armazenamento dos dados transacionais, modela-se uma base em múltiplas dimensões com objetivo de torna-la dedicada à analise. Esta base é chamada de Armazém de Dados ou DW (do inglês, Data Warehouse). O DW tem algumas caraterísticas diferentes do banco de dados transacionais como, por exemplo, não é atualizada em tempo real e apresenta redundância de valores.

Como o DW é uma base multidimensional, exploramos as combinações de variáveis para apresentação em painéis (do inglês, dashboard). Isto é possível por meio de agregações disponíveis em ferramentas de Processamento Analítico On-line ou OLAP (do inglês, Online Analytical Processing). Como explicaremos adiante, o monitoramento gráfico de atividades complementares na forma de número de solicitações por período de tempo é um exemplo de operação OLAP feita sob um DW e disponível na forma de um dashboard.

\subsection{Mineração de Dados}

Mineração de dados, por outro lado, é um conjunto de tarefas como estimação, clusterização e associação aplicadas a dados de qualquer fonte (transacional, multidimensional ou mesmo dados não-estruturado) para a descoberta de padrões [Tan et al., 2009]. Estas tarefas são geralmente implementadas por algoritmos de aprendizado de máquina ou inteligência computacional que geram modelos que podem ter como saída um dashboard ou mesmo uma dimensão do DW.

Quando o problema de aplicação for educação, chamamos de Mineração de Dados Educacionais ou EDM (do inglês, Educational Data Mining) [Romero e Ventura, 2010], [Paiva et al., 2012]. Por outro lado, se a base for textos não-estruturados, chamamos de Mineração de Textos ou TM (do inglês, Text Mining) [Berry, 2004]. E assim constituem as generalizações de áreas de aplicações em Mineração de Dados.

Neste trabalho temos características para um EDM ou TM. No entanto, o que faremos aqui é um processo envolvido no TM para a estruturação de documentos. Por tanto, não há neste projeto o uso de uma tarefa especifica de Mineração de Dados.

\subsubsection{Estruturação de documentos}

Documento é uma coleção de textos formados por um conjunto de caracteres. Cada documento tem um estilo próprio e quantidade de caracteres diferentes. E ainda, os algoritmos de aprendizado de máquina, na maioria dos casos, manipulam números e não textos. Portanto, é necessário a estruturação destes documentos.

A estruturação é iniciada por um processo de preparação de documentos que ocorre pelas seguintes etapas [Berry, 2004]:

- Análise Lexical: etapa critica da estruturação, é onde cada palavra é separadas em tokens a partir do caractere espaço. Nesta etapa também se padroniza a capitalização e é possível eliminar números e caracteres de acentuação;

- Eliminação de Stopwords: as palavras de parada são aquelas de classes gramaticais para a construção de uma frase, mas que, por outro lado, são irrelevantes para garantir a descoberta de padrão. Por exemplo, artigos, preposições, advérbios, pronomes são considerados como stopwords e devem ser removidos dos documentos; 
- Analise de Stemming: para evitar que palavras com mesmo radical sejam consideradas como sendo diferentes, nesta etapa da preparação de documentos o token deve ser reduzido ao seu radical.

Ainda pode-se considerar neste processo a etapa de Grupos Nominais, a qual é necessária para recompor em um único token, substantivos separados por uma preposição, por exemplo, "Mineração de Dados".

O resultado da preparação de documentos é uma lista única de termos que será usada como referencia no processo de representação. Neste trabalho não consideraremos a representação, sendo apenas necessárias as etapas descritas anteriormente.

\section{Metodologia Experimental e Resultados}

A base de dados para recebimento das Atividades Complementares é composta por basicamente uma tabela de modalidades (ensino, pesquisa e extensão), atividades (categorias, descrições e carga horaria), aluno (identificação, curso, semestre), solicitação (identificação do aluno, identificação da modalidade, identificação da atividade, descrição da atividade e outros atributos de avaliação do coordenador).

A partir desta base, pensamos em uma modelagem multidimensional que permitisse o levantamento dos indicadores já apresentados na introdução, como acompanhamento (numero de solicitações por categoria de atividade, modalidade, curso, semestre ou outros) e temáticas que os alunos têm se interesse por motivação do estágio ou dificuldades. Para conseguir estas respostas, pensamos em uma modelagem multimensional do tipo estrela como apresentado de maneira simplificada na Figura 1.

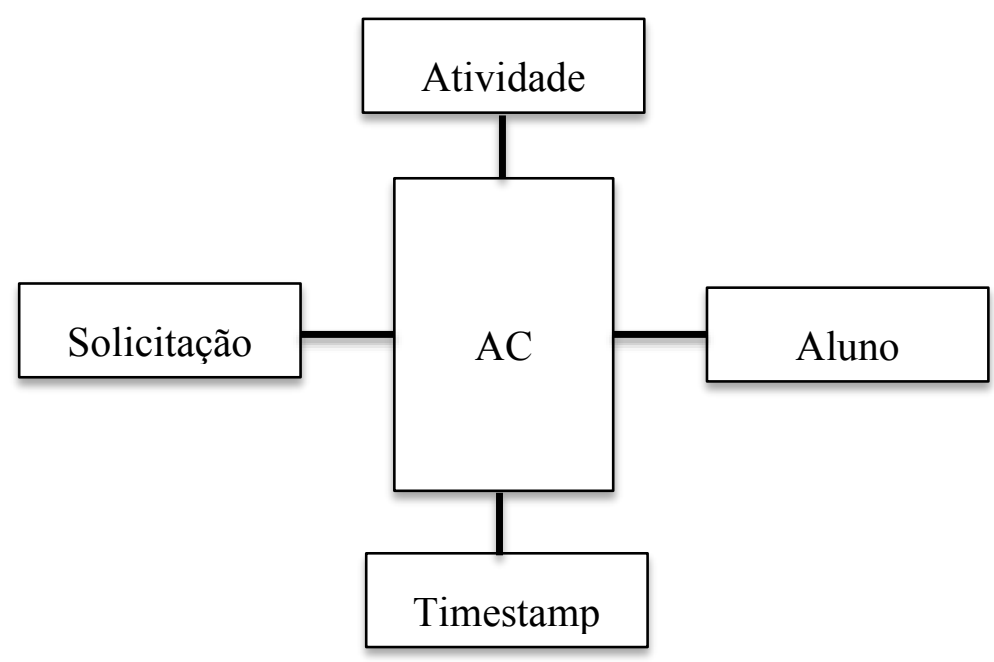

Figura 1: Modelo multimensional do tipo estrela para Atividades Complementares

No modelo proposto neste trabalho, temos como tabela fato as Atividades Complementares (AC) e dimensões, resumidamente como, Atividade, Solicitação, Aluno e Timestamp. Algumas dimensões têm atributos que podem ser decompostos, como por exemplo, a identificação do aluno. Neste atributo, os dígitos (d) que juntos totalizam 3, permitem descobrir o semestre que o aluno ingressou no curso (d) e o ano 
de ingresso (dd), e assim outros tipos de analises podem ser feitas. A dimensão solicitação, por outro lado, tem um atributo que é de texto livre, preenchido pelo aluno no momento da solicitação com até 60 caracteres. E é neste atributo que aplica-se os conceitos de estruturação de documentos, conforme explicado na Seção 4.2.1. A partir das palavras estruturadas dentro do modelo proposto na Figura 1, foi possível encontrar respostas às temáticas de maiores audiências pelos alunos (e até mesmo relacionar essa informação ao período através do relacionamento com a dimensão timestamp).

A implementação foi feita no suíte Microsoft, com o SQL Server, Analysis Services e IDE Visual Studio, todas na versão 2010. O resultado das analises será apresentado no Excel.

Os resultados serão apresentados em duas visões: coordenador e aluno. Na visão do coordenador, entre outras informações importantes, podemos verificar os assuntos mais procurados para complementação de curso, como mostrado na Figura 2. Neste resultado é possível verificar as 10 temáticas com maior número de horas que os alunos complementaram a formação. Para a coordenação, este resultado pode ser usado como indicador de assuntos que o mercado está exigindo dos alunos (muitos destes cursos são oferecidos por empresas) ou mesmo de dificuldades dos alunos (há grande procura por cursos de extensão). Este é um indicador que pode ser analisado continuamente para ter certeza de que não trata-se de um resultado momentâneo, mas talvez, por exemplo, defasagem do curso em relação à tecnologia.

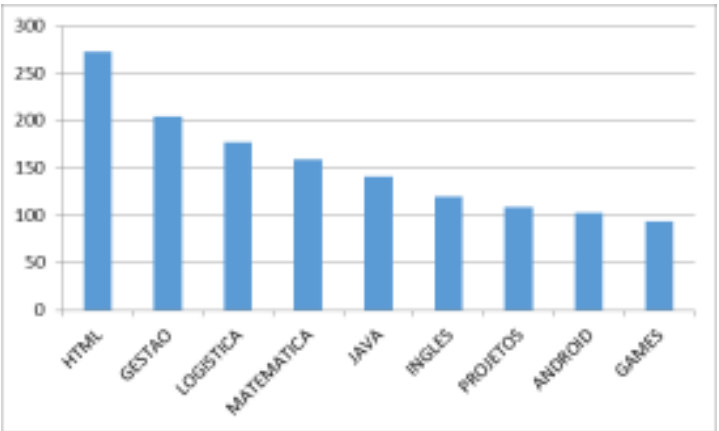

Figura 2: Total de Horas Complementares por Temáticas de Cursos de Atualização

Ainda na visão administrativa, na Figura 3 apresenta-se o total de horas realizadas por semestre. Este levantamento foi feito com dados a partir de 2008 (lembrando que as Atividades Complementares nesta UU se tornaram requisitos, a partir de 2009) até o inicio do $2^{\circ}$. Semestre de 2013. Este resultado é um importante indicador qualitativo de ações que a Unidade Universitária tem feito para incentivar a participação dos alunos na realização de Atividades Complementares. A ideia é que haja uma equalização deste gráfico ao longo dos anos. Outro. Este indicador ainda permite que a coordenação faça reflexões sobre o volume de oportunidades que estão sendo fomentadas pela UU e, até mesmo, ser uma variável para analise de evasão.

Adicionalmente ao resultado anterior, na Figura 4 temos a média de horas solicitadas por turma (complementando o que foi explicado antes, os três primeiros dígitos do número do aluno informam o semestre (3-primeiro semestre e 4-segundo semestre) e os últimos dois, o ano. Para se ter ideia, os 309 e 409 são alunos formados 
no momento da analise. Este resultado mostra um índice da quantidade de atividades que os alunos fizeram externamente a UU para se formar, sendo que as restantes são proporcionadas pela UU através de Semanas, Workshops e outros tipos de eventos extensionistas. Isto indica que o mercado está exigindo cada vez mais a complementação profissional do aluno, além do currículo básico do curso, o que deve ser monitorado, pois pode indicar defasagem do curso em relação ao mercado de trabalho.

Finalmente, para uma visão do aluno, a modelagem proposta neste trabalho permite ao aluno, entre outras informações, saber a quantidade de horas cumpridas e em quais categorias de atividades, Figura 5. Este resultado ainda auxilia o aluno no controle de atividades disponíveis no catálogo, uma vez que temos mecanismos de bloqueio para exigir que o aluno diversifique suas atividades em ensino, pesquisa e extensão. Adicionalmente a este resultado, é possível apresenta informações para o aluno se auto avaliar com a média de horas realizada pelos colegas de turma, Figura 6. Assim, uma das responsabilidades do coordenador que é lembrar os alunos desta necessidade fica dividida com o próprio aluno, pois é uma informação estará sempre apresentada a ele.

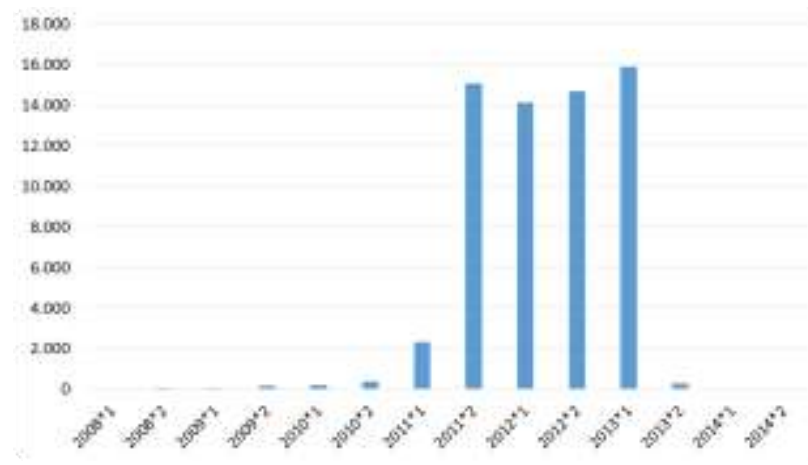

Figura 3: Total de Horas postadas por Semestre

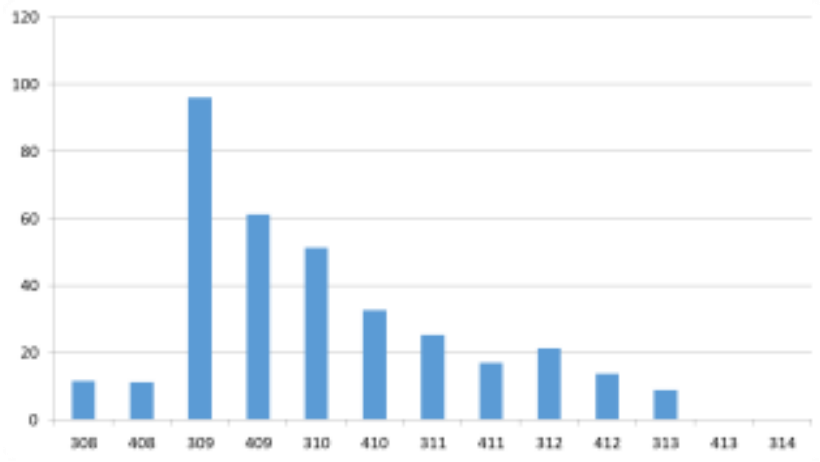

Figura 4: Média de Horas Postadas Por Turma 


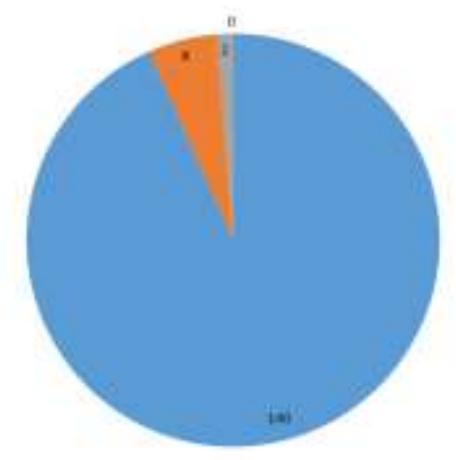

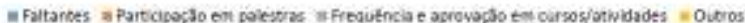

Figura 5: Balanço de Horas do Aluno

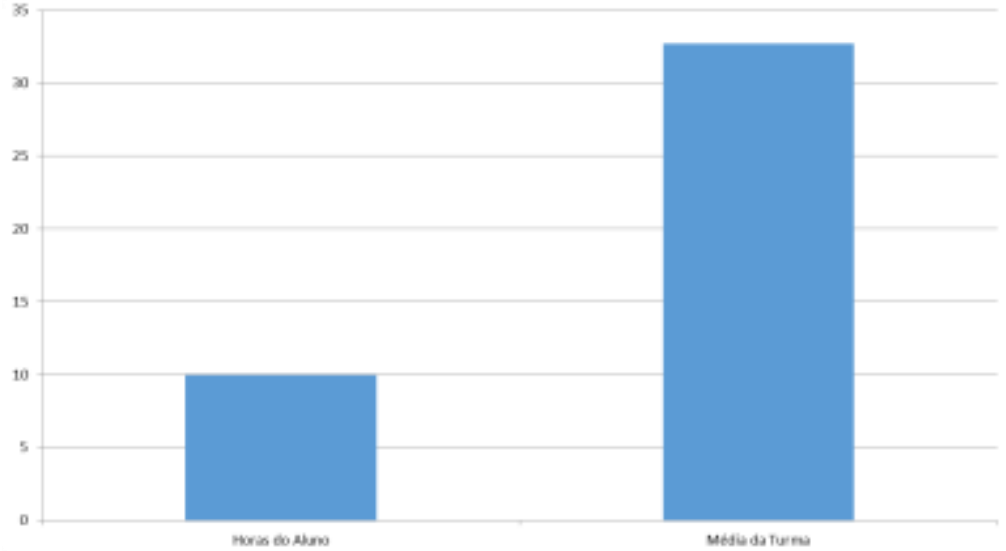

Figura 6: Comparação de Horas do Aluno com a Média da Turma.

\section{Considerações Finais}

Neste trabalho foram discutidas inicialmente as estratégias para se trabalhar as Atividades Complementares em sua função principal, qual seja, a complementação do conhecimento do aluno. Um importante mecanismo para conseguir este objetivo mais amplo é a informatização do processo de solicitação de atividades. A proposta deste trabalho foi apresentar uma modelagem analítica a partir de uma base de dados real de Atividades Complementares feitas por alunos de uma importante Faculdade de Computação.

A modelagem proposta envolveu conceitos de Inteligência de Negócios ou BI e Mineração de Dados ou DM, ambos discutidos na Seção 4 deste trabalho. De forma breve, o que fizemos foi propor uma modelagem multidimensional para um Armazém de Dados ou DW que tem uma dimensão formada por dados não-estruturados. Esta dimensão que revela as temáticas de atividades realizadas pelos alunos foi estruturada por conceitos de DM.

Os resultados das analises são apresentadas por meio de painéis (dashboards). Neste trabalho, apresentamos os painéis para uma visão da coordenação com indicadores que envolvem quantidade de horas para temáticas e turmas de alunos, as quais são subsídios para futuramente analisarmos: 
- Complementação de conceitos;

- Defasagem do Curso em relação à Tecnologia;

- Formação Pessoal;

- Monitoramento de Atividades;

- Controle de Evasão.

Como resultados adicionais, a modelagem ainda permite uma analise para o próprio aluno. Isto é, a apresentação do total de horas realizadas pelo aluno e, por outro lado, a média de horas realizada pela turma.

Futuramente, pensamos em integrar esta modelagem ao sistema de gerenciamento de atividades complementares da UU em estudo e também, a apresentação de outras analises que esta modelagem pode oferecer.

\section{Referências}

Berry, M. W. (2004). Survey of text mining. Computing Reviews, 45(9), 548.

Bittencourt, R.A., Figueiredo, O. A., "O Currículo do Curso de Engenharia de Computação da UEFS: Flexibilização e Integração Curricular”. Anais do Workshop sobre Educação em Computação, 2003.

MEC_A (2013) "Qual o conceito de Atividades Complementares?" http://portal.mec. gov.br/, Março.

MEC_B (2013) "RESOLUÇÃO $\mathrm{N}^{\circ}$ 2, DE 18 DE JUNHO DE 2007" http://portal.mec.gov.br/cne/arquivos/pdf/2007/rces002_07.pdf, Fevereiro

Paiva, R., Bittencourt I. I., Pacheco H., Silva A. P., Jacques P., Isotani S. "Mineração de dados e a gestão inteligente da aprendizagem: desafios e direcionamentos", XXXII Congresso da Sociedade Brasileira de Computação, 2012, Curitiba. Anais do DEsafIE! - I Workshop de Desafios da Computação Aplicada à Educação, 2012.

Romero, C., \& Ventura, S. (2010). Educational data mining: a review of the state of the art. Systems, Man, and Cybernetics, Part C: Applications and Reviews, IEEE Transactions on, 40(6), 601-618.

Silva, L.A., Vallim Filho, A.R.A., "Uma experiência na Utilização do Ambiente Virtual de Aprendizagem Moodle no Gerenciamento de Atividades Complementares", Congresso Brasileiro de Recursos Digitais na Educação, 2012.

Tan, P.-N.; Steinbach, M. and Kumar, V. (2009). Introdução ao Data Mining Mineração de Dados. Rio de Janeiro: Editora Ciência Moderna Ltda.

Turban, E., Sharda, R., Aronson, J. E., \& King, D. (2009). Business Intelligence: um enfoque gerencial para a inteligência do negócio. Bookman. 